\title{
Students' approaches when dealing with kinematics graphs explored by eye-tracking research method
}

\author{
Martina Kekule 1 \\ ${ }^{1}$ Department of Physics Education, Faculty of Mathematics and Physics, Charles University, Prague , Czech Republic \\ For correspondence: martina.kekule@seznam.cz
}

\begin{abstract}
The paper is focused on students graphing, particularly graphing in kinematics. The main interest of the survey was in different approaches of different students group. Particularly, groups of students who performed the test the best and the worst and groups of students who answered a task correctly or incorrectly. Altogether 26 students participated in the survey from high schools and colleges. 7 tasks mostly adopted from TUGK test by Beichner (1994) were used for testing. Eye tracker research method was used and data were analysed qualitatively based on heat maps of number of fixations. We identified different numbers of fixations distributed among areas of tasks for the groups of students. Especially in most cases "Best" students did not show the same pattern like students who answered a task correctly. This indicates some general different approaches which are used by the "Best" group students. Particularly, they more frequently fixated instructions in contrast to the rest areas of the graph. They tended to judge the whole curve of a graph. Students who answered correctly or belonged to the group "Worst" tend to fixate more often the same point within the area of the task. These results suggest some hypothesis for further quantitative research.
\end{abstract}

Keywords: graphs, kinematics, physics, students, eye tracking, heat maps, qualitative survey

\section{Introduction}

The paper is focused on graphs in physics education. Why should teachers teach graphs? And why in physics classes? The answer to these two questions is quite easy. Media such as TV, newspapers or internet often use graphs for data presentation. Therefore, it is obvious that the ability to work with graphs is or will be necessary for everybody. From this point of view, teachers should teach students to use graphs above all as a communication tool. During study, students should gain especially these skills: how to get information from a graph and how to show data, information by means of graphs. The motivation why in general graphs should be taught is quite clear. However, why should teachers focus on graphs also in physics classes? "The primary goal of science education across the EU should be to educate students both about the major explanations of the material world that science offers and about the way science works" (Osborne and Dillon, 2008). The way science work of course include graphing, because "graph construction and interpretation are very important because they are an integral part of experimentation, the heart of science" (Beichner, 1994). Typical misconcepcion when students deal with graphs like with pictures or photographs of real situation is very common when students are busy with graphs of kinematic variables. That is why graphing in kinematic can reveal the misconception and enable introduce a graph as an abstract chart.

The main purpose of the presented qualitative study is to compare approaches to solving the presented tasks of different students group. Especially, the main interest is in comparison between students who performed the whole test the best (or the worst) and the ones who answered correctly just a task. The topic is part of experts/novices paradigma which is in physics education research transformed to comparision between students who either performed a test well or solve presented problem right (for example see Madsen et al., 2012). 


\section{Methodology}

For obtaining answers to the research questions tasks included in the Test of Understanding Graphs in Kinematics developed by Beichner (1994) were administered to students and their way of solution by eyetracking method was tracked.

\section{Participants}

There were 25 participants in the study ( 8 women, 15 men). Most of the participants were graduate students (18) of science (focused on physics, chemistry or geology), 12 of the students attended the first year of their study (age 20). 4 students were students at higher secondary level of education (3 of them of age 18). One participant was scientist at the physics department (age 33). To sum up description of participants, there is more than half of student aged 18-20 who did not take advanced course in graduate physics.

\section{Apparatus}

Eyetracker by Tobii was used, particularly TX300 with frequency $300 \mathrm{~Hz}$, which has accuracy less than $0.5^{\circ}$ of visual angle. The infrared camera was placed under the 23 -inch screen of the stimulus PC. Stimuli were presented as .pdf document. Participant's eyes were positioned at a distance of approximately $70 \mathrm{~cm}$ from the center of the screen. A five point calibration and validation procedure was used before start of experiment. Eye movements were recorded by Tobii Studio 3.2 and for identification of fixations inbuilt IVT filter were used. Eye movement was classified as a saccade when eye's velocity exceeded $30^{\circ} / \mathrm{s}$. Minimum fixation duration was set up $60 \mathrm{~ms}$. An extra mouse and keyboard were connected to the stimulus PC by which participants handle the PC.

\section{Procedure}

Firstly, each participant fulfilled a questionnaire about their learning preferences (adopted from LSI questionnaire) and completed a kinematic task with a graph. Then he or she took part in an individual eyetracking session lasting about 15 minutes. This included calibration process, instruction session about the following tasks. Before each task short instruction about what task will follow was presented so that students had enough time to relax before they started to solve a new task. During the session students were ask for thinking aloud when it is needed and the sessions were recorder by video camera. After the sessions students were asked to provide verbal retrospective report.

\section{Materials}

Altogether students solve ten tasks concerning graphs of kinematics variable. 5 tasks were adopted from the Test of Understanding Graphs in Kinematics developed by Beichner (1994). Particularly, tasks were these misconceptions were identified:

- kinematic variable confusion (task no. $3,5,8$ ),

- slope/height confusion (task no. 4 and 6).

The latter misconception was identified when students solve a task concerning comparison speed of objects which position-time graphs are presented (task no. 1). The task was adopted from (McDermott, et al. 1987). The last task proofed students' ideas about abstract conception of a graph (task no. 7). 3 tasks were omitted from further analysis due to imprecise formulation of a tasks. Within paper questionnaire students solve an additional problem, where students sometimes show typical "graphs as a picture" misconception. The task was used in my previous survey in Czech Republic (Kekule, 2010). Detailed description of tasks including presented graphs is available at Appendix I.

\section{Analysis}

For understanding research issue mainly qualitative research methodology were used. So that for analysis of eye movement data mainly heat maps were used. Heat maps summarize large quantities of data, which can be further judge visually. For creating heat maps Tobii Studio 3.2 were used. Heat maps were calculated based on identified fixations (in contrast to raw data), particularly number of fixation was taken into account. Number of fixation on an area shows students' interest in the area. So it is relevant variable for finding out different approaches of different students' groups. During the 
first phase, pixels are coloured by the same colour if they belongs to the same fixation. During the second phase the heat map image is created smoother by "adding colour values to all the points within a certain distance of the fixation location" (Tobii Manual). This is ensured by using Cubic Hermite Spline polynomial (t2(3-2t)), where the total kernel is of 100 pixels (Tobii Manual).

For our analysis we divide students into groups. The firstly, we identified students with the best and the worst total score of the all tasks including additional task in paper questionnaire. Group "Best" include students with total score more than $80 \% .6$ students met the demand. Characteristics of the students: 1 female/5 males, 4 aged 23-25/2 aged 18-20, 2 study physics. Group "Worst" include students with total score less than $30 \% .4$ students met the demand. Characteristics of the group: 1 female/3 males, all aged 18-20, none study physics. Secondly, we identified students which solved the particular task correctly or vice versa. These groups of students vary according to a task.

\section{Results}

For comparison among different students' groups qualitative analysis is provided, particularly based on heat maps. Heat maps of groups "Best" and "Worst" were created and of students who answered a task correctly or incorrectly were provided as well. Detail specification of the groups see part

"Analysis" above. The heat maps are presented in the Table 1.

Table 1. Heat maps for tasks of different students groups. Groups of students who got more than 80 $\%$ / less than $30 \%$ of the total score of the whole test. Groups of students who answered a task in/correctly.

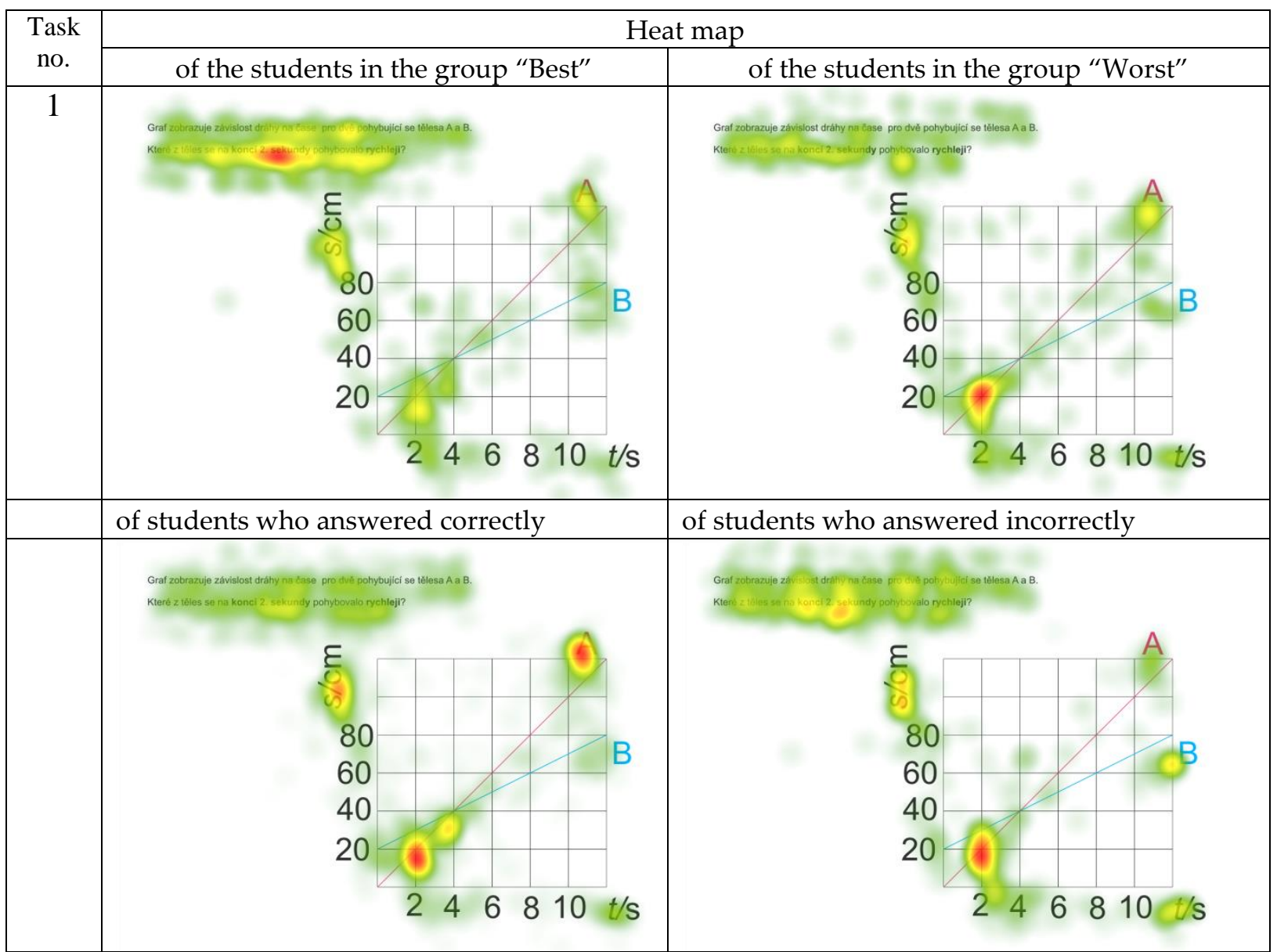




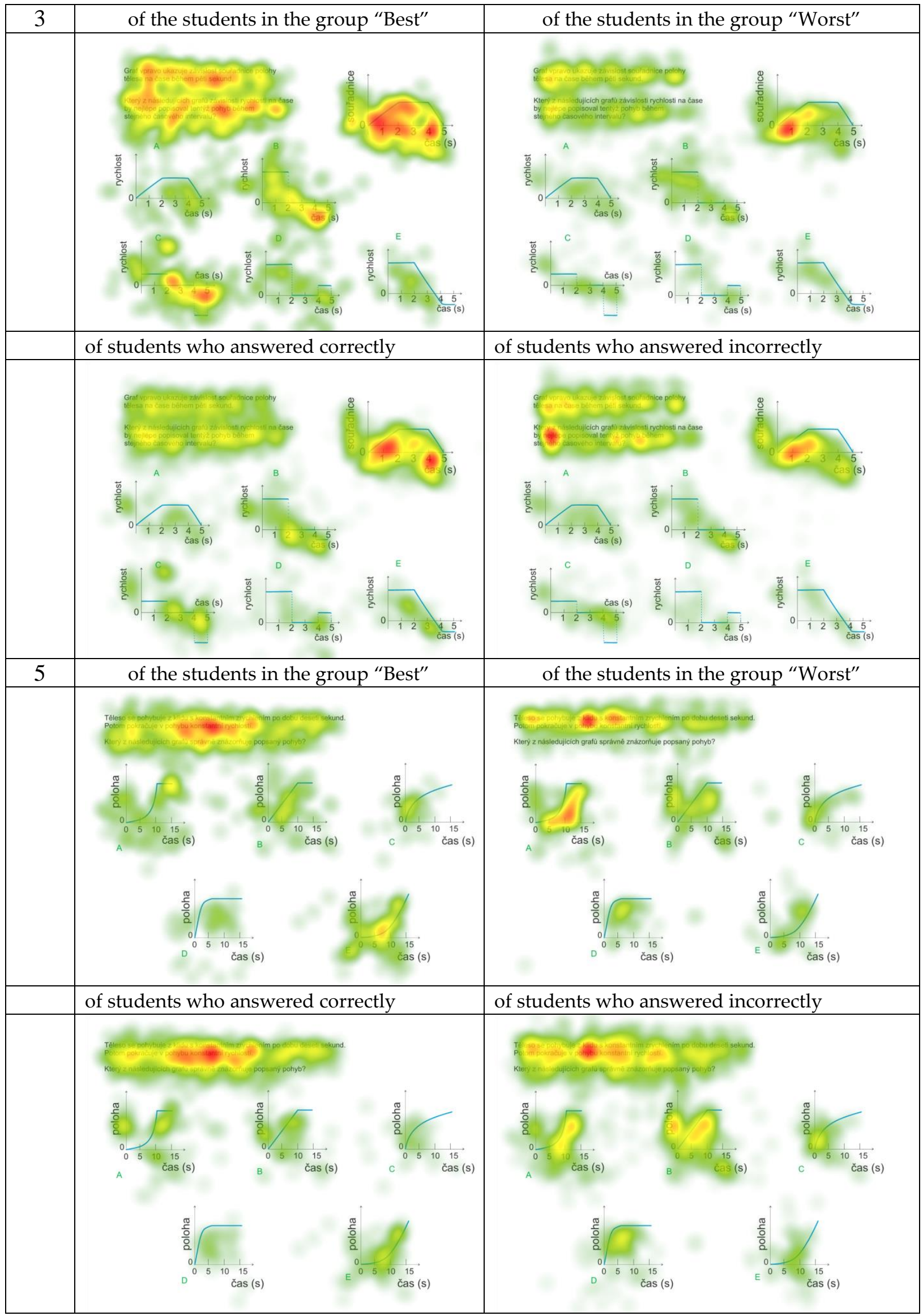




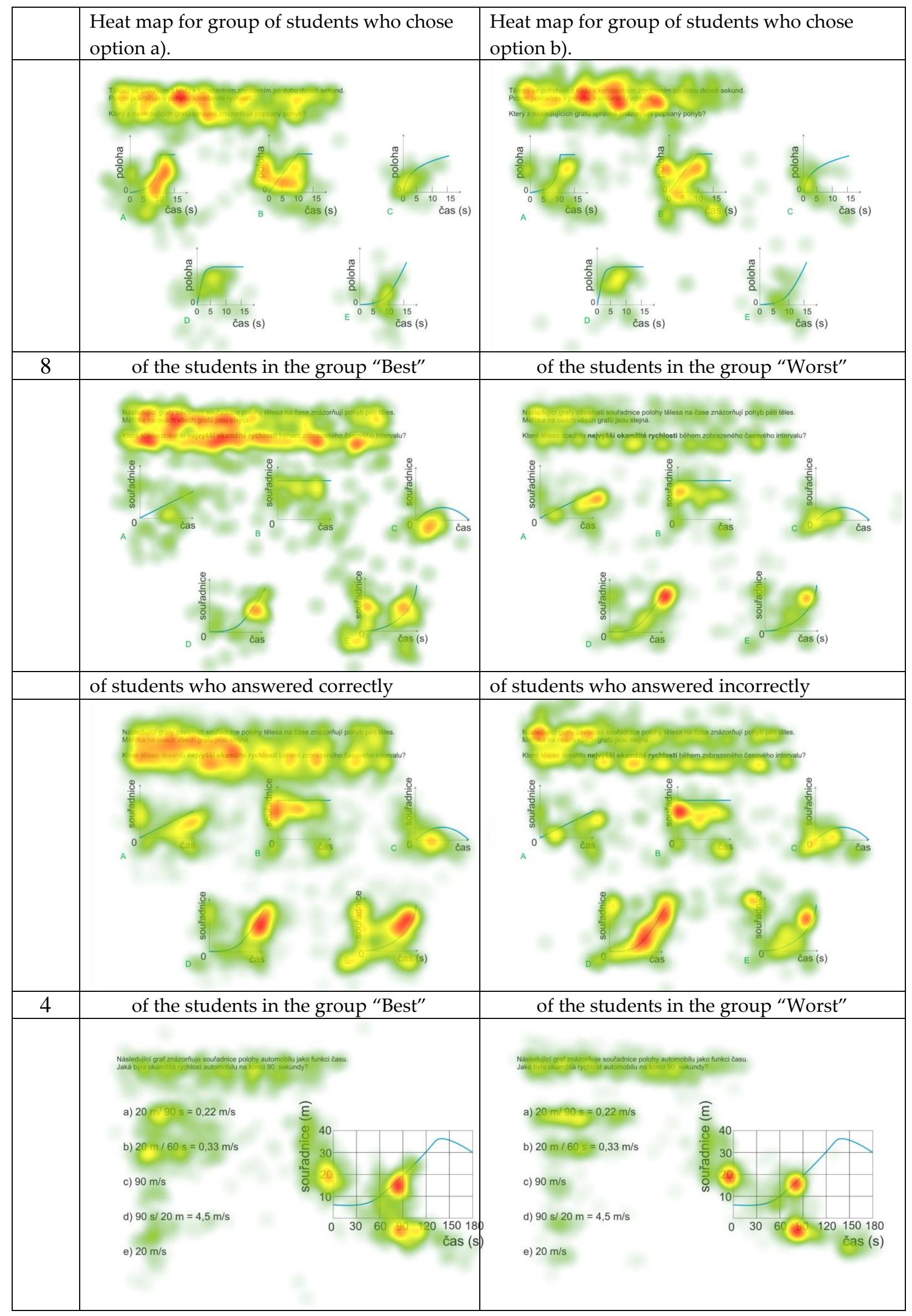




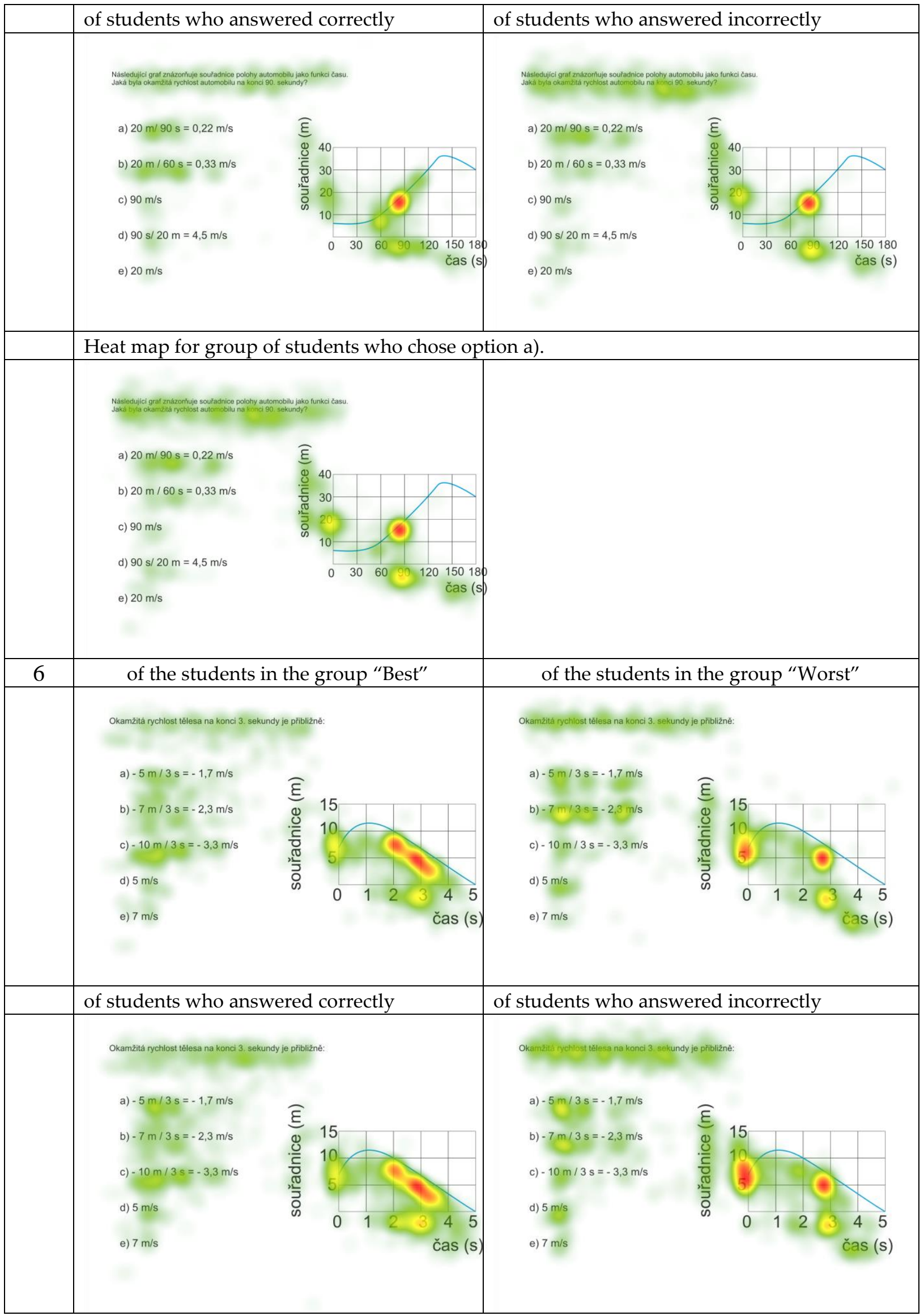




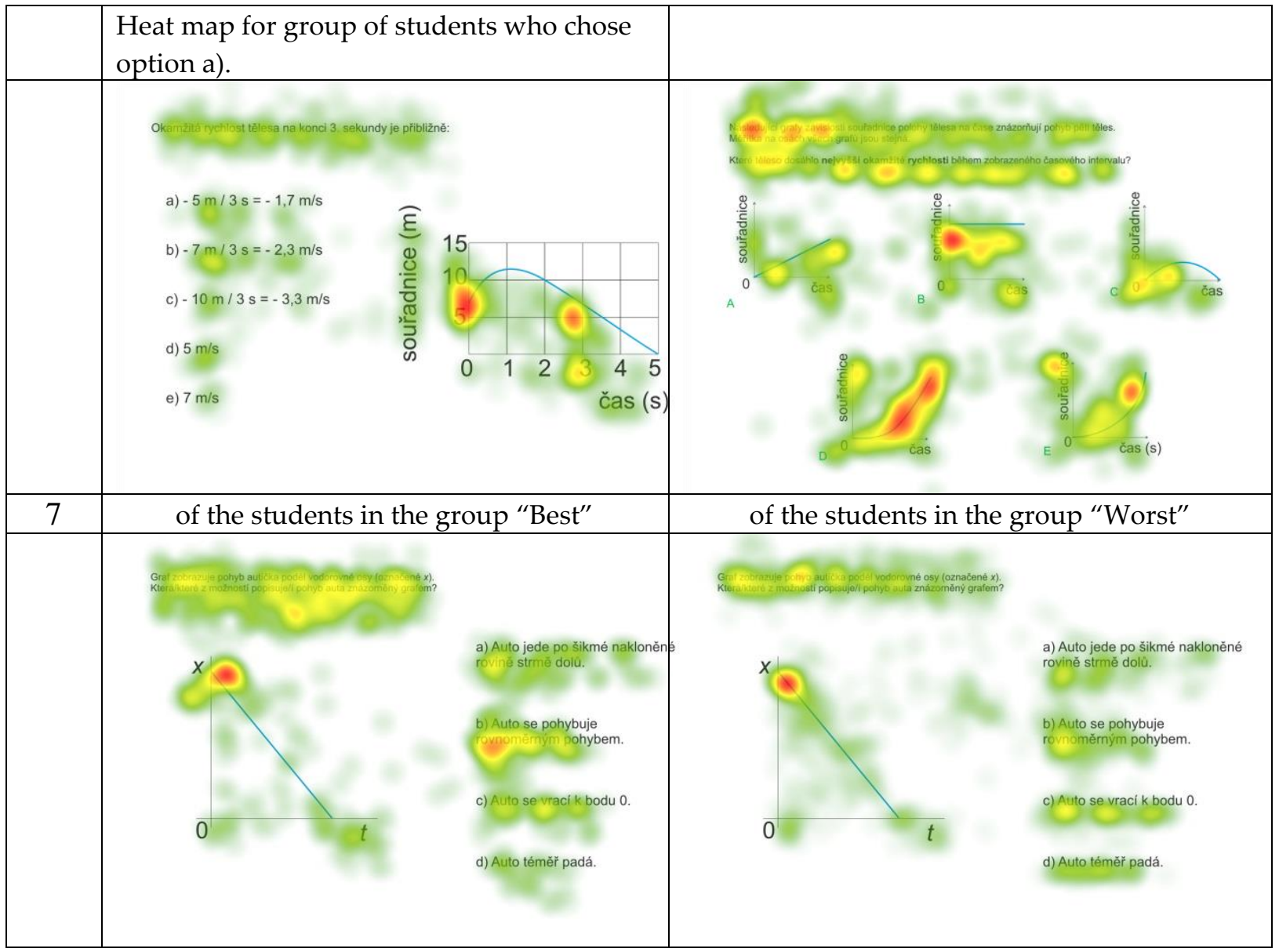

Note: One or two students from the group "Best" solved task no. 1, 4 and 5 incorrectly. If we omitted their recordings, we get very similar heat maps as it is shown in Figure 1.
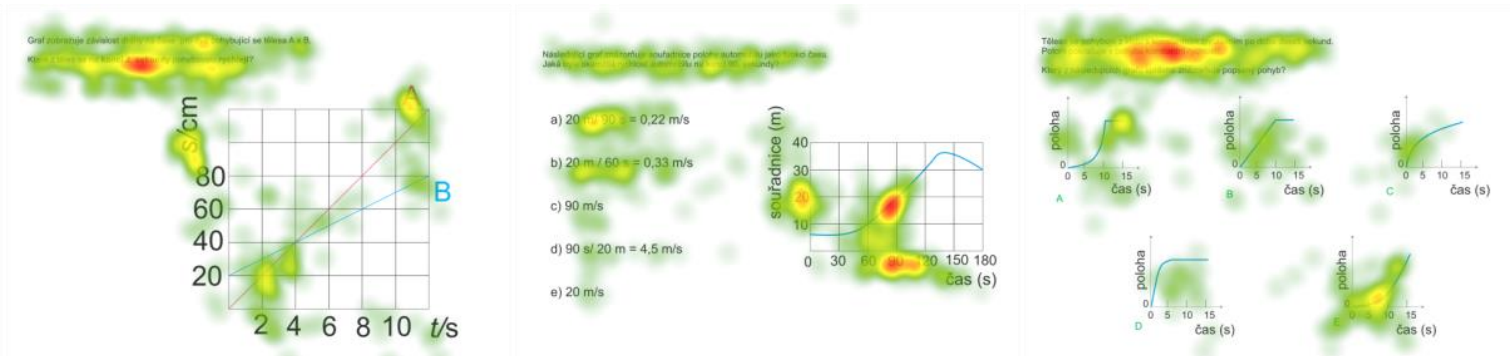

Figure 1. Corrected heat maps (incorrect answers omitted) of the group "Best" for task no. 1, 4 and 5.

Emphasize of the analysis is on

1. Identification of differences between groups "Best" and "Worst".

2. Identification of differences between groups "Best" and of students who answered correctly.

3. Identification of differences between groups of students who answered correctly and incorrectly.

Task no. 1

1. The most frequently fixated area for group "Best" is the key information in instruction (i.e. we are interested at moment about the end of $2^{\text {nd }}$ second). The most frequently fixated area for group "Worst" is value of the graph at the end of $2^{\text {nd }}$ second.

2. Similar to the point 1 .

3. Very similar, except fixation of letters A and B. Each group fixated more frequently the letter the answer was provided. 
Task no. 3

1. Group "Best" most frequently fixated the whole graph presented within instruction in contrast to the group "Worst" who were the most interested in the origin of the graph. The group "Best" were obviously interested in options b) and c). They most frequently fixated instructions.

2. The group of students who answered correctly the most frequently fixated edges of the graph curve. The less frequently they were interested in the instructions and options B and C. It is probable, that interpretation of the graph curve were for the students more difficult than for students in the group "Best".

3. A heat map of students who answered incorrectly is very similar to the group "Worst". Task no. 5

1. The group "Best" slightly more frequently fixated instructions. The group "Worst" was much more interested in option A, particularly in the whole curve of the graph.

2. Students who answered correctly show less frequent fixation of the right graph in comparison to the other fixated areas.

3. Student who answered incorrectly fixated much more often options A and B. If we split the group of students according to their answer (whether they chose option a) or b)), heat maps Task no. 8 not surprisingly shows again the most frequent fixations on options $\mathrm{A}$ and $\mathrm{B}$.

1. The group "Best" more frequently fixated instruction. They were interested in the whole area of the right graph.

2. Students who answered correctly more often fixacet the last two options, especially the ends of graph curves. Less frequently they fixated instructions.

3. Student who answered incorrectly were the most interested in last two graphs (D, E) as well. However, the more frequently fixated the whole curve of the graphs in contrast to students who provide the right answer.

Task no. 4 and 6

Both tasks demands the same skills, the only difference is in the orientation of the slope of the graph.

1. The group "Best"in both cases shows fixations following rather the curve of the graph. Especially this is more salient in the task no. 6 where the negative slope means not so typical task for students and so more attention was needed. The "Worst" group fixated rather isolated point which is in agreement with their provided answer.

2. Students who answered correctly fixated less numbers of axis which they wanted to extract. They much more frequently were interested in the graph curve, especially in the value at the time $90 \mathrm{~s}$ for task no. 4.

3. Student who answered incorrectly in contrast to those who did right do not show interest in graph values at times 60 and $120 \mathrm{~s}$ for task no. 4 .

\section{Conclusion}

The paper is focused on students graphing, particularly graphing in kinematics. The main interest of the survey was in different approaches of different students group. Particularly, groups of students who performed the test the best and the worst and groups of students who answered a task correctly or incorrectly. Eye tracker research method was used and data were analysed qualitatively based on heat maps of number of fixations. We identified different number of fixations distributed among areas of tasks for the groups of students. Especially in most cases "Best" students did not show the same pattern like students who answered a task correctly. This indicates some general different approaches which are used by the "Best" group students. Particularly, they more frequently fixated instructions in contrast to the rest areas of the graph. They tended to judge the whole curve of a graph. Students who answered correctly or belonged to the group "Worst" tend to fixate more often the same point within the area of the task. These results suggest some hypothesis for further quantitative research. 


\section{References}

Beichner, R. (1994). Testing students interpretation of kinematic graphs. Am. J. Phys. 62 (8), 750-762

McDermott, L.C., Rosenquist, M.L., van Zee, E.H. (1987). Student difficulties in connecting graphs from kinematice. Am. J. Phys. 55 (6), 503-513

Kekule, M. Graphs in Physics Education. In GIREP 2008 International Conference Physics Curriculum Design and Development and Validation. (ed.) Nicosia : The Learning Science Group, Department of Educational Studies, University of Cyprus, 2010. 978-9963-689-21-7

Madsen, A. et al. (2012). Differences in visual attention between thos who correctly and incorrectly answer physics problems. Physical Review Special Topics - PER. DOI: 10.1103/PhysRevSTPER.8.010122

Tobii Manual. User manual - Tobii Studio. Manual Version 3.2 Rev A. 11/2012. Tobii Technology AB. Available from http:// www.tobii.com

\section{Appendix I - Description of tasks}

The appendix presents description of tasks which were used for students testing. As it is stated in the paper above, half of the task is adopted from test by Beichner (1994) and so original tasks are available there.

\section{Task 1}

\begin{tabular}{|l|l|l|l|l|}
\hline Students should determine which of the objects was moving \\
faster at time about 2 s. Position-time graph. (From \\
McDermott et al., 1987)
\end{tabular}

Task 3

Given position - time graph (see picture). Students should choose from five velocity - time graphs which show the same motion. (From Beichner 1994, task no. 11)

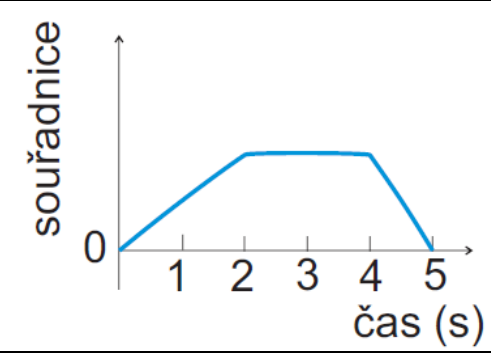

Task 4

Given position - time graph (see picture). Students should determine speed at the end of $90^{\text {th }}$ second. They could choose from five options. (From Beichner 1994, task no. 6, numbers adjusted).

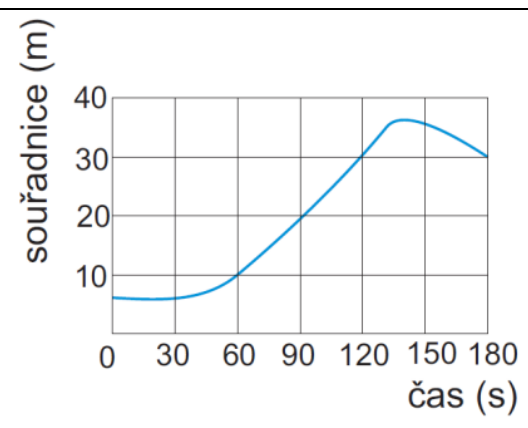

Task 5

Given description of a motion. Students should choose from five position - time graphs which describe the motion. (From Beichner 1994, task no. 9) 
Task 6

Given position - time graph (see picture). Students should determine speed at the end of $3^{\text {rd }}$ second. They could choose from five options. (From Beichner 1994, task no. 17, numbers adjusted).

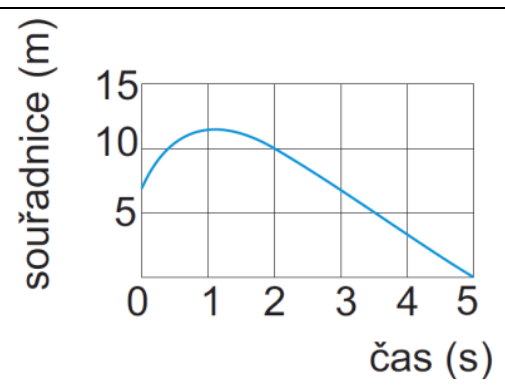

Task 7

Given position - time graph (see picture). Students should choose from five options which describe the motion.

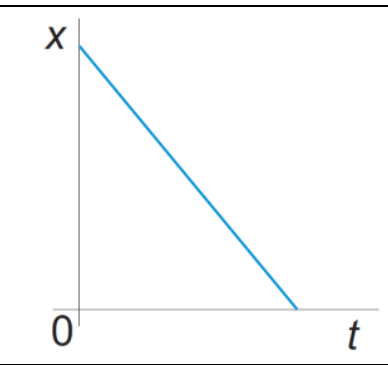

Task 8

Five position-time graphs are given. Students should choose the option where an object reach the highest speed. From Beichner 1994, task no. 13).

Task within paper questionnaire

Given horizontal coordinate-time graphs. Students should choose which graph describes the relation between time and horizontal coordinate of a projectile motion for horizontal/vertical/general launch. (From Kekule, 2010)

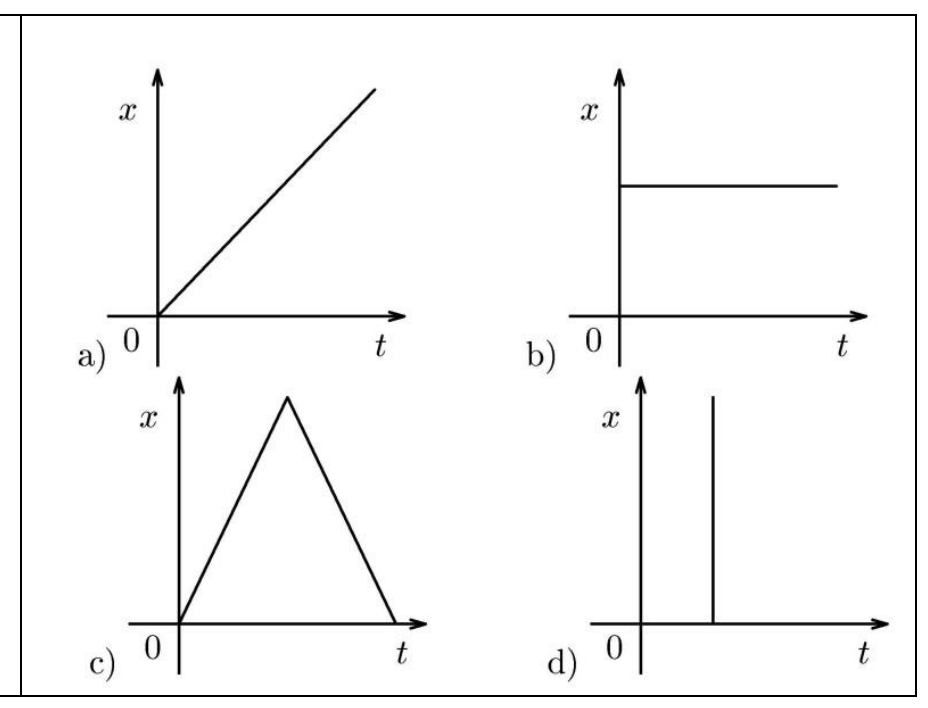

